\title{
Article \\ A Partial Information Decomposition Based on Causal Tensors
}

\author{
David Sigtermans $^{1 *}$ \\ 1 ASML \\ * Correspondence: david.sigtermans@asml.com \\ Received: date; Accepted: date; Published: date
}

\begin{abstract}
We propose a partial information decomposition based on the newly introduced framework of causal tensors, i.e., multilinear stochastic maps that transform source data into destination data. The innovation that causal tensors introduce is that the framework allows for an exact expression of an indirect association in terms of the constituting, direct associations. This is not possible when expressing associations only in measures like mutual information or transfer entropy. Instead of a priori expressing associations in terms of mutual information or transfer entropy, the a posteriori expression of associations in these terms results in an intuitive definition of a nonnegative and left monotonic redundancy, which also meets the identity property. Our proposed redundancy satisfies the three axioms introduced by Williams and Beer. Symmetry and self-redundancy axioms follow directly from our definition. The data processing inequality ensures that the monotonicity axiom is satisfied. Because causal tensors can describe both mutual information as transfer entropy, the partial information decomposition applies to both measures. Results show that the decomposition closely resembles the decomposition of other another approach that expresses associations in terms of mutual information a posteriori. A negative synergistic term could indicate that there is an unobserved common cause.
\end{abstract}

Keywords: information theory; causal inference; causal tensors; transfer entropy; partial information decomposition; transmission path; left monotonicity; identity property; unobserved common cause

\section{Introduction}

The ability to decompose information within a multivariate system, i.e., systems comprising over two random variables, allows us to diagnose behavior of these systems. Mutual information, the information measure used in information theory [1], does not lead to a satisfactory decomposition. For example, the widely used "Interaction Information" [2] can have negative values, which is counterintuitive. An alternative approach is offered via "partial information decomposition" [3]. Total information is written as the sum of nonnegative information components. The definition of these nonnegative information components is still an open problem however, as summarized in [4].

In this article we contribute to this discussion by showing that a partial information decomposition in nonnegative contributors follows naturally from the framework of causal tensors, i.e., the set of transition probability matrices. For mutual information, the tensor can be represented by a matrix. The tensor comprises several transition probability matrices in the case of transfer entropy. Information theory [1] models the association between data as transmission of source data towards a destination via a communication channel. A channel is characterized by its probability transition matrix [5]. The association between the source data and the destination data is the mutual information (MI). If data is transmitted from a source to a mediator and from there to the destination, i.e., via a transmission path comprising three or more nodes, the mutual information between the source and destination can not be expressed in terms of the mutual informations between the source and mediator and the MI between the mediator and destination. Using the causal tensors however, the causal tensor of the resulting communication channel between source and destination is a function of the constituting communication channels along the transmission path. 
Instead of a priori expressing associations in terms of mutual information or transfer entropy, we propose to use a posteriori expression of associations in these information measures. In doing so, an intuitive definition of redundancy and related, unique information arises.

\section{Materials and Methods}

Because causal tensors as a representation for the communication channel plays a central role, a short summary is given

\subsection{Causal tensors}

In information theory, the data are realizations of random variables representing stationary ergodic processes [1]. Because the data comprise elements from a finite alphabet, we can describe an outcome using the value, i.e., alphabet symbol, or using the index, assuming a fixed, e.g., lexicographic, order of the alphabet elements. In this article we use the latter.

The communication channel transforms the probability mass function (pmf) of the source data in the pmf of the destination data via a linear mapping. With $p^{j}$ representing the $j^{\text {th }}$ element of the destination pmf, and $p^{i}$ representing the $i^{\text {th }}$ element of the source pmf, the relation between source and destination is given by

$$
p^{j}=\sum_{i} p^{i} A_{i}^{j}
$$

The elements of the tensor $\mathcal{A}$, i.e., $A_{i}^{j}$, represent the transition probabilities $A_{i}^{j}=$ $p\left(j^{\text {th }}\right.$ destination symbol $i^{\text {th }}$ source symbol $)$. A communication channel is the conceptual implementation of the Law of Total Probability [6]. This immediately implies that the source pmf can be reconstructed from the destination pmf: $p^{i}=\sum_{j} p^{j} A_{j}^{\ddagger i}$. The $\ddagger$ indicates that the source pmf is reconstructed. This is relevant in case a source is distinguishable from a destination, i.e., when transfer entropy is used. The mutual between the source data, generated by process $X$, and the destination data, generated by the process $Y$, is expressed as

$$
I(X, Y)=\sum_{i, j} p^{i j} \log _{2}\left[\frac{A_{i}^{j}}{p^{j}}\right] .
$$

Equation 1 is equivalent to standard expression for mutual information, $I(X, Y)=$ $\sum_{x \in \mathcal{X}, y \in \mathcal{Y}} p(x, y) \log _{2}[p(y \mid x) / p(y)]$ (see, for example, [5]). This can be seen by switching from the index notation to the notation in alphabet elements by replacing the transition probability matrix elements $A_{i}^{j}$ with the conditional probability $p(y \mid x)$, the joint probability $p^{i j}$ with $p(x, y)$, and the output probability $p^{j}$ with $p(y)$. The variable $x$ is selected from alphabet $\mathcal{X}$, and the variable $y$ is selected from the alphabet $\mathcal{Y}$ respectively.

Transfer entropy [7] is an information theoretical implementation of "Wieners principle of causality" [8]: a "cause" combined with the past of the "effect" predicts the effect better than that the "effect" predicts itself. It was proven that with a slight modification of the original proposed transfer entropy (TE), it fully complies with Wieners principle of causality [9]. Transfer entropy is the measure of association for data transmission via a network of communication channels with an inverse multiplexer topology (see Figure 1a). This becomes apparent when transfer entropy is written as

$$
T E_{X \rightarrow Y}=\sum_{\psi_{g}^{-} \in \mathcal{Y}^{\ell}} p\left(\psi_{g}^{-}\right) \sum_{\substack{\mathbf{x}^{-} \in \mathcal{X}^{m} \\ y \in \mathcal{Y}}} p\left(\mathbf{x}^{-}, y \mid \mathbf{y}^{-}=\psi_{g}^{-}\right) \log _{2}\left[\frac{\left.p\left(y \mid \mathbf{x}^{-}, \mathbf{y}^{-}=\psi_{g}^{-}\right)\right)}{p\left(y \mid \mathbf{y}^{-}=\psi_{g}^{-}\right)}\right]
$$

with $\mathbf{x}^{-}$representing the cause selecting symbols from the alphabet $\mathcal{X}^{m}, \mathbf{y}^{-}$representing the past of the effect, $\psi_{g}^{-}$equals the $g^{\text {th }}$ alphabet element from the alphabet $\mathcal{Y}^{\ell}$, and $y$ representing the effect. 
It is assumed that $Y$ is a Markov process of order $\ell$, and that the "cause" consists of $m$ subsequent symbols.

Transition probability matrices can be associated with this inverse multiplexer channel. There are $\left|\mathcal{Y}^{\ell}\right|$ sub-channels, where $|\cdot|$ indicates the cardinality of the alphabet. Per sub-channel, the communication channel is identical to a communication channel giving rise to $\mathrm{MI}$ as the measure of association, therefore, the elements of the $g^{\text {th }}$ sub-channel of the causal tensor $\mathcal{A}$ are given by $A_{g i}^{j}$. Like MI, transfer entropy can be expressed in terms of the tensor:

$$
T E_{X \rightarrow Y}=\sum_{g, i, j} p^{i j g} \log _{2}\left[\frac{A_{g i}^{j}}{p_{g}^{j}}\right] .
$$

Mutual information also results from transmission over an inverse multiplexer, an inverse multiplexer comprising one sub-channel.

Definition 1. Mutual information is the measure of association from data transmission via a single-channel inverse multiplexer. Transfer entropy is the measure of association from data transmission via a multi-channel inverse multiplexer.

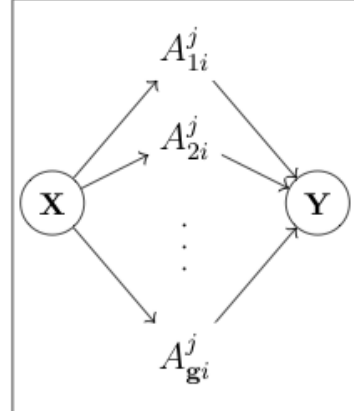

(a)

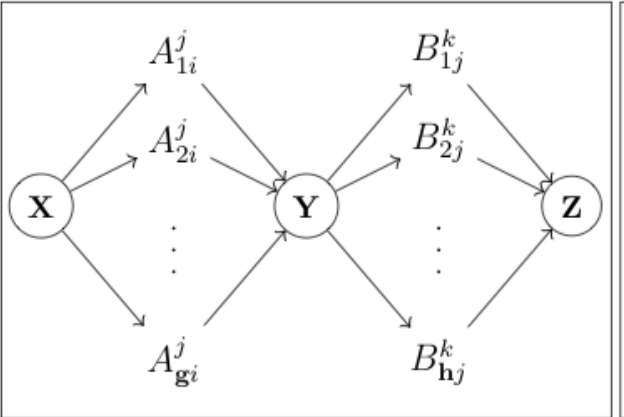

(b)

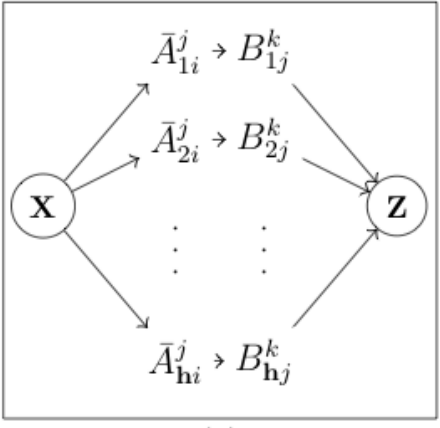

(c)

Figure 1. (a) The inverse multiplexer representing the communication network between $X$ and $Y$. Source data is partitioned on the past of the effect, indicated by index $g$, and transmitted via the related communication channel. (b) The inverse multiplexers representing the transmission path $\{x, y, z\}$. Index $h$ is related to the past of the $z$. (c) An equivalent representation network communication channels representing the transmission path $\{x, y, z\}$. The causal tensor elements $\bar{A}_{h i}^{j}$ equals $\sum_{g} p_{h i}^{g} A_{g i}^{j}$. Per "sub-channel", the resulting causal tensor equals $\sum_{j} \bar{A}_{h i}^{j} B_{h j}^{k}$.

\subsubsection{Causal Tensors of a Cascade and Transmission Paths}

A system comprising more than one random variable can be expressed as a graph in which the nodes represent the random variables, and the edges represent associations. The edges are undirected when MI is used as a measure of association and directed when TE is used. This gives rise to transmission paths.

Definition 2. A transmission path, or path in short, is defined as the sequence in which nodes have been used to transmit the data. The source is defined as the first variable in the path, the destination is the last variable in the path. A path is denoted as $\left\{\right.$ source, mediator $_{1}, \cdots$, mediatior $_{n}$, destination $\}$.

The association between a source and destination of a path comprising over two nodes, i.e., a cascade of (direct) paths, cannot be expressed in terms of the association constituting direct paths when MI or TE are used. However, the following theorem can be proven [10]: 
Theorem 1. The causal tensor of a cascade of paths can be expressed in terms of the causal tensors of the constituting direct paths.

For example, assume that the tensor elements for the path $\{x, y\}$ are given by $A_{g i}^{j}$, with $g$ the index for the past of the effect $(y)$, and the tensor elements for the path $\{x, y\}$ are given by $B_{h j}^{k}$, with $h$ the index for the past of the effect $(z)$. The causal tensor $\mathcal{T}$ for the path $\{x, y, z\}$ equals

$$
\mathcal{T}\{x, y, z\}=\sum_{g, j} p_{h i}^{g} A_{g i}^{j} B_{h j}^{k} .
$$

Figure $1 \mathrm{~b}$ depicts the transmission of data over two inverse multiplexers in series, resulting in Eq.(2). The term $\sum_{g} p_{h i}^{g} A_{g i}^{j}$ can be interpreted as the weighted sum of the causal tensors of the sub-channels of the first direct path, $\{x, y\}$, and it evaluates to a causal tensor $\bar{A}_{h i}^{j}$ [10]. Transmission of data over a cascade of multi-channel inverse multiplexers is equivalent to the transmission of data over a parallel set of cascades of single-channel inverse multiplexers, i.e., results applicable to MI also apply to TE. For this reason, we can restrict ourselves, without loss of generality, to MI. The mutual information for the path $\{x, y, z\}$, can be expressed in the tensor elements $\mathcal{T}\{x, y, z\}=\sum_{j} A_{i}^{j} B_{j}^{k}$,

$$
I\{x, y, z\}=\sum_{i, j, k} p^{i j k} \log _{2}\left[\frac{\sum_{j} A_{i}^{j} B_{j}^{k}}{p^{k}}\right] .
$$

\subsubsection{Indirect Associations and No Associations}

There are two underlying reasons to delete an edge in a graph: ( $i$ ) the association it represents is indirect, or (ii) there is no association at all. These two reasons can be distinguished using causal tensors. First, because the causal tensor of a cascade can be determined exactly from the constituting causal tensors, causal tensors can differentiate between direct and indirect associations [10]. Second, if there is no association between two nodes at all, the causal tensor represents a communication channel that cannot transmit any information. Here the transition probability matrix has identical rows, e.g., $\forall i \neq f: A_{i}^{j}=A_{f}^{j}$.

Proposition 1. If the association between two nodes is indirect, or if the association does not exist, the direct path does not exist.

For example, the path $\{x, z\}$ does not exist when the graph $X \rightarrow Y \rightarrow Z$ is the ground truth.

\subsection{Partial Information Decomposition}

The partial information decomposition framework of Williams and Beer [3] allows for a decomposition of the total information in nonnegative unique, redundant, and synergistic information components. The unique information $\mathcal{U}(Y ; Z)$ represents information in $Z$ only provided by $Y$ and not by $X$. The redundant information $\mathcal{R}(X, Y ; Z)$ represents the information in $Z$ provided by both $X$ and $Y$. The synergistic information $\mathcal{S}(X, Y ; Z)$ represents information in $Z$ that results via interaction between $X$ and $Y$. The relations between these information components for a system comprising three variables are given by the following set of equations,

$$
\begin{aligned}
I(X, Y ; Z) & =\mathcal{U}(Y ; Z)+\mathcal{U}(X ; Z)+\mathcal{R}(X, Y ; Z)+\mathcal{S}(X, Y ; Z), \\
I(Y ; Z) & =\mathcal{U}(Y ; Z)+\mathcal{R}(X, Y ; Z), \\
I(X ; Z) & =\mathcal{U}(X ; Z)+\mathcal{R}(X, Y ; Z) .
\end{aligned}
$$


Williams and Beer propose three redundancy related axioms: (i) Symmetry: redundancy does not change when sources are permuted, e.g., $R(Z ; X, Y)=R(Z ; Y, X)$. (ii) Self-Redundancy: for a single source, the redundancy equals the mutual information between the source and the destination, e.g., $R(Z ; X)=I(Z ; X)$. (iii) Monotonicity: the redundancy does not increase when a new source is added, e.g., $R(Z ; X) \geq R(Z ; X, Y)$.

\subsubsection{Redundancy, Indirect Paths and the Data Processing Inequality}

If the association between two nodes is indirect, no direct path exists. Source data is transmitted to the mediator node, which stores, possibly copies, possibly modifies, and possibly enriches the received information with information from this mediator node, after which it is transmitted again towards the next mediator node or destination node. This consideration leads to the following proposition:

Proposition 2. Unique information can only result from data transmission via a direct path. Redundant information is the consequence of data transmission via an indirect path.

A direct consequence of this proposition is that in case the chain $X \rightarrow Y \rightarrow Z$ is the ground truth, no unique information is shared between $X$ and $Z$, or stated otherwise, all information shared between $X$ and $Z$ is redundant. Another immediate consequence of this proposition is that in case of the XOR example from [11], there is neither unique, nor redundant information shared between the sources and the destination: all information shared is synergistic.

In a fully connected three-node system, there are two source nodes transmitting data to the destination node, and per source node, there is one indirect path between that source node and the destination node. All indirect paths fall in two categories: (i) the indirect path includes the direct path between a specific source and destination, and (ii) the indirect path does not include the direct path between a specific source and destination. For the first category, the Data Processing Inequality (DPI) [5] is directly applicable. Data Processing Inequality states that processing of data can never increase the amount of information. For the path $\{x, y, z\}$ this means that $I\{x, y, z\} \leq \min [I\{x, y\}, I\{y, z\}]$. Via this path the redundant information from $X$ is transmitted. For the path $\{y, x, z\}$ this means that $I\{y, x, z\} \leq \min [I\{y, x\}, I\{x, z\}]$. Via this path the redundant information from $Y$ is transmitted. Based on this example, the following definition of redundancy is proposed:

Definition 3. Redundant information shared between a specific set of sources with respect to a destination is defined as the weakest of all indirect paths that: (1) contain all the sources, (2) start with a source and ends at the destination, and (3) do not contain non-existing paths.

Because the redundancy in this definition equals a mutual information, the proposed redundancy is per definition nonnegative. This definition of redundancy also satisfies the three axioms introduced in [3].

Sketch of Proof of Symmetry. Because of the definition of redundancy, all indirect paths representing all permutations of sources are compared. The order of the sources in the redundancy expression is therefore irrelevant.

Sketch of Proof of Self-Redundancy. Assume that one source is a copy of the other source, e.g., $Y=X$. This means that for the causal tensors describing the mapping between the sources and destination, $\mathcal{B}$ and $\mathcal{C}: \mathcal{B}=\mathcal{C}$. Per definition $R(X, X ; Z)=I\{x, x, z\}$. The causal tensor for the path $\{x, x\}, \mathcal{T}\{x, x\}$, equals the Kronecker delta $\delta_{i}^{j}: \delta_{1}^{j}=0$ unless $i=j$ in which case $\delta_{i}^{j}=1$. Therefore, the causal tensor for the path $\{x, x, z\}$ equals $\mathcal{B}$, i.e., $I\{x, x, z\}=I(X ; Z)$.

Sketch of Proof of Monotonicity. Because of the definition of redundancy, adding more sources increases the number of nodes within the indirect path. As per Data Processing inequality, the adding sources can never increase the redundancy. 
Apart from these three axioms, Bertschinger et al. [12] and Harder et al. [13] proposed other properties. The proposed redundancy measure satisfies both the "left monotonicity" property and the "identity property".

\subsubsection{Left Monotonicity Property}

Left monotonicity captures the intuition that if $X$ and $Y$ share some information about $Z_{1}$, "then at least the same amount of information is available to reduce the uncertainty about the joint outcome" of $Z_{1}$ and $Z_{2}[12]$ :

$$
R\left(Z_{1} ; Y, X\right) \leq R\left(Z_{1} Z_{2} ; Y, X\right) .
$$

For readability, the proof of the left monotonic property has been moved to Appendix A.

\subsubsection{Identity Property}

The intuition behind the identity property [13] is that if the destination is a join of the inputs, the redundancy equals the mutual information of inputs, i.e.,

$$
R(X Y ; X, Y)=I(Y ; X)
$$

with $X Y=X \cup Y$. In Appendix B we prove that the proposed redundancy property satisfies the identity property.

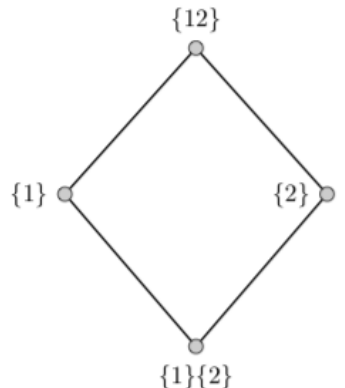

(a)

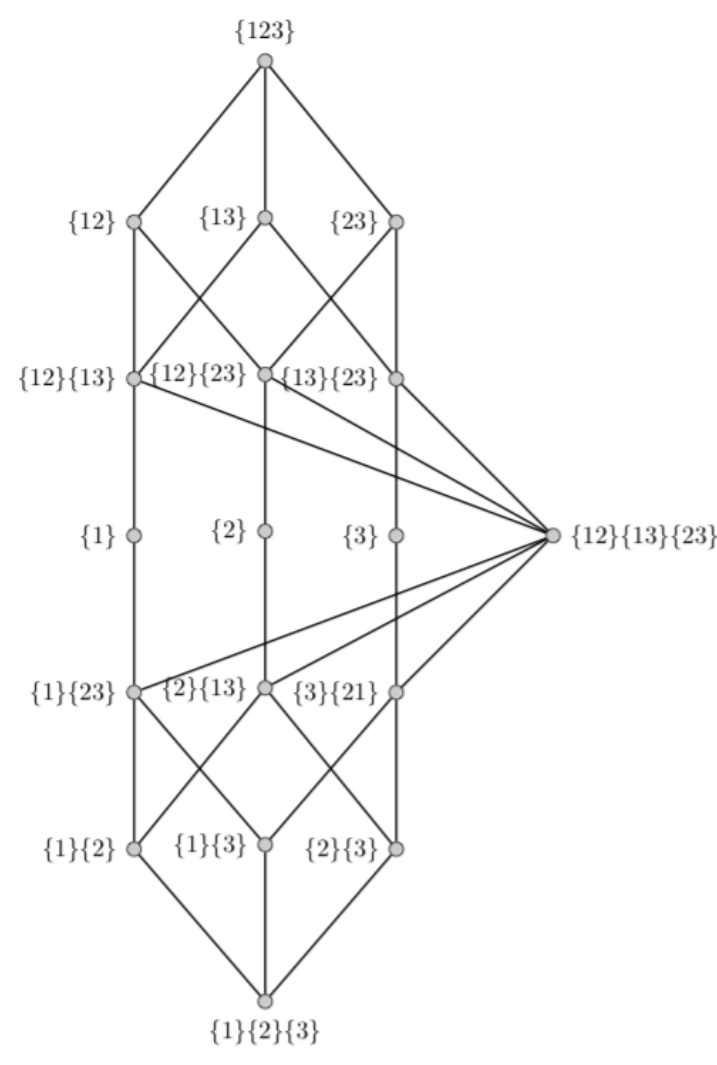

(b)

Figure 2. The redundancy lattice [3] for: a) two sources, and (b) three sources. In case two lattice nodes are connected, then the redundancy related to the highest lattice node (in position) is greater than or equal to the redundancy of the lower lattice node. 
For a three-node system, the proposed redundancy gives rise to an identical redundancy lattice introduced by [3], and shown in Figure 2. Because of the DPI there is a natural ordering in terms of (self-) redundancy:

- $R(X, X ; Z) \geq R(X, Y ; Z)$,

- $R(Y, Y ; Z) \geq R(X, Y ; Z)$,

- $R(X Y, X Y) \geq R(Y, Y ; Z)$,

- $R(X Y, X Y) \geq R(X, X ; Z)$,

with $X Y=X \cup Y$. The last two relations are a consequence of the fact that the tensor of the path $\{x, z\}$ can be expressed as a tensor contraction of the tensors related to the paths $\{x y, z\}$ and $\{x, y\}$, and that the tensor of the path $\{y, z\}$ can be expressed as a tensor contraction involving the tensors related to the path $\{x y, z\}$ and $\{y, x\}[10]$.

\subsubsection{Unique Information}

Unique information for a three-node system is defined by Eq.(4) and Eq.(5). Using the proposed redundancy, the resulting unique information is nonnegative because of the DPI. The proof is straightforward and left to the reader. The unique information defined in this fashion, fully matches our intuition. This can demonstrated by applying it to the fully connected three-node system. The information in $Z$ results from information transmitted via the paths $I\{x, y, z\}$ and $I\{y, x, z\}$. The redundant information $\mathcal{R}(X, Y ; Z)$ equals

$$
\mathcal{R}(X, Y ; Z)=\min [I\{x, y, z\}, I\{y, x, z\}] .
$$

The causal tensor elements of the paths $I\{y, x, z\}$ and $I\{x, y, z\}$ are given by $\sum_{i} A_{j}^{\ddagger i} C_{i}^{k}$ and $\sum_{j} A_{i}^{j} B_{j}^{k}$ respectively. Using Eq.(4) the unique information we get

$$
\mathcal{U}(Y ; Z)=\max \left[\sum_{i, j, k} p^{i j k} \log _{2}\left[\frac{B_{j}^{k}}{\sum_{m} A_{j}^{\ddagger m} C_{m}^{k}}\right], \sum_{i, j, k} p^{i j k} \log _{2}\left[\frac{B_{j}^{k}}{\sum_{\ell} A_{i}^{\ell} B_{\ell}^{k}}\right]\right] .
$$

The first sum is a measure for the divergence between the direct path $\{y, z\}$ and the indirect path $\{y, x, z\}$. If the association between $Y$ and $Z$ is indirect, this sum evaluates to zero. This also implies that a direct path between $Y$ and $Z$ does not exist. The second sum is an indication how much $Y$ differs from an exact copy of $X$. If $Y$ is an exact copy, this term evaluates to zero: no unique information is shared between $Y$ and $Z$ because all information was already shared via $X$.

\subsubsection{Relationship of Proposed Redundancy with Redundancy Lattice}

As shown earlier, the proposed redundancy for two sources matches the redundancy lattice derived in [3]. With three sources, this is also the case. The lattice nodes are indicated as $\left\{X_{1}\right\}\{\cdots\}\left\{X_{n}\right\}$ (see Figure 2). The redundancy related to the lattice node equals $R\left(X_{1}, \cdots, X_{n} ; Z\right)$, i.e., the minimum overall mutual informations of the indirect paths ending in $Z$ and containing all permutations of the sources $X_{1}, \cdots, X_{n}$. This means that a lattice node gives a description of all individual sources involved. Because of the DPI, the redundancy associated with a lattice node must be less than or equal to the redundancy of lattice nodes comprising only a subset of the sources. For example, $R(1,23 ; Z) \leq R(1 ; Z)$, where 23 indicates the join of the sources 2 and 3 . When this is combined with the order suggested by the redundancy lattice for two sources, e.g., $R(12 ; Z) \geq R(1 ; Z)$, the proposed definition fully complies with the order implied by the redundancy lattice.

\section{Results}

In this section the behavior of the proposed partial information decomposition we start with investigating its behavior with respect the conceptual issue related to, $I_{\min }$, the original redundancy 
measure used in [3]: $I_{\text {min }}$ does not distinguish between "same information" or "the same amount of information".

\subsection{Two Bit Copy Problem}

A conceptual problem with the redundancy measure used in [3], $I_{m i n}$, is illustrated with the so called "two-bit copy problem". For two independent and identically distributed binary variables $X$ and $Y$, the destination $Z$ is a copy of these two variables: $Z=(X, Y)$. It can be shown that $I_{\min }(Z ;\{1\}\{2\})=$ 1 bit [14]. The problem lies in the fact that there is no overlap between the information of both variables: the result does not match out intuition, $I_{\min }$ seems to overestimate the redundancy. All indirect paths

Table 1. (Distribution for the "two-bit copy problem".

\begin{tabular}{cccc}
\hline$X$ & $Y$ & $Z$ & $p(Z)$ \\
\hline 0 & 0 & $(0,0)$ & $\frac{1}{4}$ \\
0 & 1 & $(0,1)$ & $\frac{1}{4}$ \\
1 & 0 & $(1,0)$ & $\frac{1}{4}$ \\
1 & 1 & $(1,1)$ & $\frac{1}{4}$ \\
\hline
\end{tabular}

contain the paths between $X$ and $Y$. Using Table 1 , the causal tensor for the path $\{x, y\}, \mathcal{A}$, can be determined:

$$
\mathcal{A}=\left(\begin{array}{ll}
\frac{1}{2} & \frac{1}{2} \\
\frac{1}{2} & \frac{1}{2}
\end{array}\right) .
$$

The tensor related to the path $\{y, x\}, \mathcal{A}^{\ddagger}$, equals $\mathcal{A}$. This implies that no information can be transmitted via the paths between $X$ and $Y$, i.e., $R(X, Y ; Z)=0$. The proposed redundancy does not suffer from this conceptual issue, because it satisfies the identity property.

\subsection{Negative Synergistic Contributions Due to Unobserved Common Causes?}

A result of the proposed definitions is that an unobserved common cause can lead to a negative synergistic contribution. In Table 2 an example is given of such a system. If the common cause $\tilde{Z}$ is observed, the path $\{x, y\}$ does not. However, if the common cause is unobserved, there is no reason to state that the path is nonexistent. The reader can confirm that the resulting synergistic term is negative because the redundancy $R(Z ; X, Y)$ equals 0.0271 bit and the interaction information, $I(X ; Y)-I(X ; Y \mid Z)[2]$, equals 0.1226 bit.

Table 2. Example of a negative synergistic component when there is an unobserved common cause. (a) Data set comprising three parameters. (b) Hidden common cause. $X=\tilde{Z}_{1}, Y=\tilde{Z}_{1}$ OR $\tilde{Z}_{2}$, and $Z=\tilde{Z}_{1} \mathrm{ANDZ}_{2}$.

\begin{tabular}{ccccc}
\hline & (a) Data set & & & (b) Common cause \\
\hline$X$ & $Y$ & $Z$ & $p$ & $\tilde{Z}=\tilde{Z}_{1} \tilde{Z}_{2}$ \\
\hline 0 & 0 & 0 & $\frac{1}{4}$ & 00 \\
0 & 1 & 0 & $\frac{1}{4}$ & 01 \\
1 & 1 & 0 & $\frac{1}{4}$ & 10 \\
1 & 1 & 1 & $\frac{1}{4}$ & 11 \\
\hline
\end{tabular}

It is currently unclear if a negative synergistic term always implies that there is an unobserved common cause. Further research is needed to answer that question.

\subsection{Local Positivity}

The proposed redundancy satisfies the left monotonicity property and the identity property both properties. Bertschinger et al. proved however that the left monotonicity property and the identity 
property are incompatible with nonnegative partial information components for systems comprising over three nodes [15], i.e., the local positivity property is violated. However, essential to the proposed redundancy is that it arises due to the existence of indirect paths. If there are no indirect paths, there is no redundancy. All information is, as per our definition, unique and/or synergistic. When we apply this definition to the system used in the proof, a system comprising four nodes, $X, Y, X$ XOR $Y(X \oplus Y)$, and $(X, Y, X \oplus Y)$, the system does not contain indirect paths (see Figure 3). As per our definition, $(X, Y, X \oplus Y)$ only contains nonnegative unique information.

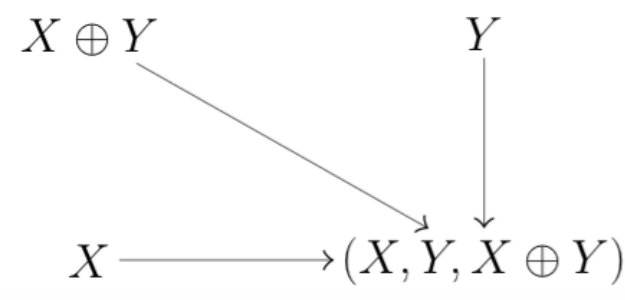

Figure 3. The graph for the system used to prove that the left monotonicity property and the identity property are incompatible with the local positivity property.

We need to stress here that this consequence of our definition does not disprove Bertschingers conclusion. It only shows that with a different definition, for this case, local positivity is guaranteed.

\subsection{Dyadic and Triadic Systems}

Next we apply the proposed method to the two data sets from Table 3. Although these sets have different underlying dependency structures, they apparently have the same statistical structure [16].

Table 3. Two systems, both comprising three random variables with identical joint probabilities per combination of the random variables. The underlying structures are very different, which can be seen when the variables are represented in two bits, e.g., the binary expansion for $X=3$ equals $X_{0} X_{1}=11$. (a) For the dyadic (pair-wise) set, $X_{0}=Y_{1}, Y_{0}=Z_{1}$, and $Z_{0}=X_{1}$. (b) For the triadic (three-way) set, $X_{0}+Y_{0}+Z_{0} \bmod 2$, and $X_{1}+Y_{1}+Z_{1}$.

\begin{tabular}{cccccccc}
\hline & (a) Dyadic & & \multicolumn{5}{c}{ (b) Triadic } \\
\hline$X$ & $Y$ & $Z$ & $p$ & $X$ & $Y$ & $Z$ & $p$ \\
\hline 0 & 0 & 0 & $\frac{1}{8}$ & 0 & 0 & 0 & $\frac{1}{8}$ \\
0 & 2 & 1 & $\frac{1}{8}$ & 1 & 1 & 1 & $\frac{1}{8}$ \\
1 & 0 & 2 & $\frac{1}{8}$ & 0 & 2 & 2 & $\frac{1}{8}$ \\
1 & 2 & 3 & $\frac{1}{8}$ & 1 & 3 & 3 & $\frac{1}{8}$ \\
2 & 1 & 0 & $\frac{1}{8}$ & 2 & 0 & 2 & $\frac{1}{8}$ \\
2 & 3 & 1 & $\frac{1}{8}$ & 3 & 1 & 3 & $\frac{1}{8}$ \\
3 & 1 & 2 & $\frac{1}{8}$ & 2 & 2 & 0 & $\frac{1}{8}$ \\
3 & 3 & 3 & $\frac{1}{8}$ & 3 & 3 & 1 & $\frac{1}{8}$ \\
\hline
\end{tabular}

For the dyadic set, the causal tensors are given by:

$$
\mathcal{A}=\left(\begin{array}{cccc}
\frac{1}{2} & 0 & \frac{1}{2} & 0 \\
\frac{1}{2} & 0 & \frac{1}{2} & 0 \\
0 & \frac{1}{2} & 0 & \frac{1}{2} \\
0 & \frac{1}{2} & 0 & \frac{1}{2}
\end{array}\right), \mathcal{C}=\left(\begin{array}{cccc}
\frac{1}{2} & \frac{1}{2} & 0 & 0 \\
0 & 0 & \frac{1}{2} & \frac{1}{2} \\
\frac{1}{2} & \frac{1}{2} & 0 & 0 \\
0 & 0 & \frac{1}{2} & \frac{1}{2}
\end{array}\right)
$$

$\mathcal{B}=\mathcal{A}, \mathcal{A}^{\ddagger}=\mathcal{C}, \mathcal{B}^{\ddagger}=\mathcal{C}$, and $\mathcal{C}^{\ddagger}=\mathcal{A}$. Because no relation is the result of a cascade, e.g., $B_{j}^{k} \neq \sum_{i} A_{j}^{\ddagger i} C_{i}^{k}$, the structure is that of an undirected triangle. Lets assume we are interested in the partial information 
decomposition of the total information in Z. Because $\forall i, k: \sum_{j} A_{i}^{j} B_{j}^{k}=\frac{1}{4}$, the redundant information equals zero. For the triadic set, the causal tensors are given by:

$$
\mathcal{A}=\left(\begin{array}{cccc}
\frac{1}{2} & 0 & \frac{1}{2} & 0 \\
0 & \frac{1}{2} & 0 & \frac{1}{2} \\
\frac{1}{2} & 0 & \frac{1}{2} & 0 \\
0 & \frac{1}{2} & 0 & \frac{1}{2}
\end{array}\right),
$$

$\mathcal{B}=\mathcal{A}, \mathcal{C}=\mathcal{A}, \mathcal{A}^{\ddagger}=\mathcal{A}, \mathcal{B}^{\ddagger}=\mathcal{A}$, and $\mathcal{C}^{\ddagger}=\mathcal{A}$. Here, the ground structure is that of a chain because any relation results from a cascade, e.g., $C_{i}^{k}=\sum_{j} A_{i}^{j} B_{j}^{k}$. To compare the partial information decomposition of the triadic set, we have to investigate the set of the chains $X \rightarrow Y \rightarrow Z$ and $Y \rightarrow X \rightarrow Z$. By definition, $Z$ does not contain unique information from $X$, nor does it contain any unique information from $Y$.

This example shows that the difference in underlying structure is reflected in two ways. First, the graphs related to the dyadic set and the triadic set are different (a triangle versus a chain). Second, for the dyadic set there is no redundant information and no synergistic information, because the total information in $\mathrm{Z}$ can not exceed 2 bit. In the triadic set, $\mathrm{Z}$ contains only redundant and synergistic information.

\subsection{Comparison with Other Measures}

To get an idea about the behavior of the proposed redundancy measure compared three other measures: (i) the earlier mentioned redundancy measure $I_{\text {min }}$, (ii) $I_{b r o j a}$, the redundancy measure proposed in [17], and (iii) the redundancy based on Pointwise Common Change in Surprisal, $I_{C C S}$ [14]. For a description of the examples we refer to [14]. The proposed redundancy measure is represented as $I_{\triangle}$.

Table 4. PID for 5A.

\begin{tabular}{ccccc}
\hline Lattice Node & $I_{\partial}\left(I_{\text {min }}\right)$ & $I_{\partial}\left(I_{\text {broja }}\right)$ & $I_{\partial}\left(I_{C C S}\right)$ & $I_{\partial}\left(I_{\triangle}\right)$ \\
\hline$\{12\}$ & 0.3333 & 0 & 0.1383 & 0.1383 \\
$\{2\}$ & 0.3333 & 0.6666 & 0.5283 & 0.5283 \\
$\{1\}$ & 0.3333 & 0.6666 & 0.5283 & 0.5283 \\
$\{1\}\{2\}$ & 0.5850 & 0.2516 & 0.3900 & 0.3900 \\
\hline
\end{tabular}

Table 5. PID for 5в.

\begin{tabular}{ccccc}
\hline Lattice Node & $I_{\partial}\left(I_{\text {min }}\right)$ & $I_{\partial}\left(I_{\text {broja }}\right)$ & $I_{\partial}\left(I_{C C S}\right)$ & $I_{\partial}\left(I_{\triangle}\right)$ \\
\hline$\{12\}$ & 0.5 & 0 & 0 & 0 \\
$\{2\}$ & 0.5 & 1 & 1 & 1 \\
$\{1\}$ & 0 & 0.5 & 0.5 & 0.5 \\
$\{1\}\{2\}$ & 0.5 & 0 & 0 & 0 \\
\hline
\end{tabular}

Table 6. PID for 5C.

\begin{tabular}{ccccc}
\hline Lattice Node & $I_{\partial}\left(I_{\text {min }}\right)$ & $I_{\partial}\left(I_{\text {broja }}\right)$ & $I_{\partial}\left(I_{C C S}\right)$ & $I_{\partial}\left(I_{\triangle}\right)$ \\
\hline$\{12\}$ & 0.67 & 0.67 & 0.67 & 0.67 \\
$\{2\}$ & 0.25 & 0.25 & 0.25 & 0.25 \\
$\{1\}$ & 0 & 0 & 0 & 0 \\
$\{1\}\{2\}$ & 0 & 0 & 0 & 0 \\
\hline
\end{tabular}


Table 7. PID for REDUCEDOR.

\begin{tabular}{ccccc}
\hline Lattice Node & $I_{\partial}\left(I_{\text {min }}\right)$ & $I_{\partial}\left(I_{\text {broja }}\right)$ & $I_{\partial}\left(I_{C C S}\right)$ & $I_{\partial}\left(I_{\triangle}\right)$ \\
\hline$\{12\}$ & 0.69 & 0.69 & 0.38 & 0.40 \\
$\{2\}$ & 0 & 0 & 0.31 & 0.29 \\
$\{1\}$ & 0 & 0 & 0.31 & 0.29 \\
$\{1\}\{2\}$ & 0.31 & 0.31 & 0 & 0.02 \\
\hline
\end{tabular}

Table 8. PID for XOR.

\begin{tabular}{ccccc}
\hline Lattice Node & $I_{\partial}\left(I_{\text {min }}\right)$ & $I_{\partial}\left(I_{\text {broja }}\right)$ & $I_{\partial}\left(I_{C C S}\right)$ & $I_{\partial}\left(I_{\triangle}\right)$ \\
\hline$\{12\}$ & 1 & 1 & 1 & 1 \\
$\{2\}$ & 0 & 0 & 0 & 0 \\
$\{1\}$ & 0 & 0 & 0 & 0 \\
$\{1\}\{2\}$ & 0 & 0 & 0 & 0 \\
\hline
\end{tabular}

An interesting difference between other proposed methods and our proposed method is that there is no redundancy in the AND/OR case.

Table 9. PID for AND/OR.

\begin{tabular}{ccccc}
\hline Lattice Node & $I_{\partial}\left(I_{\text {min }}\right)$ & $I_{\partial}\left(I_{\text {broja }}\right)$ & $I_{\partial}\left(I_{C C S}\right)$ & $I_{\partial}\left(I_{\triangle}\right)$ \\
\hline$\{12\}$ & 0.5 & 0.5 & 0.29 & 0.19 \\
$\{2\}$ & 0 & 0 & 0.21 & 0.31 \\
$\{1\}$ & 0 & 0 & 0.21 & 0.31 \\
$\{1\}\{2\}$ & 0.31 & 0.31 & 0.10 & 0 \\
\hline
\end{tabular}

Table 10. PID for SUM.

\begin{tabular}{ccccc}
\hline Lattice Node & $I_{\partial}\left(I_{\text {min }}\right)$ & $I_{\partial}\left(I_{\text {broja }}\right)$ & $I_{\partial}\left(I_{C C S}\right)$ & $I_{\partial}\left(I_{\triangle}\right)$ \\
\hline$\{12\}$ & 1 & 1 & 0.5 & 0.5 \\
$\{2\}$ & 0 & 0 & 0.5 & 0.5 \\
$\{1\}$ & 0 & 0 & 0.5 & 0.5 \\
$\{1\}\{2\}$ & 0.5 & 0.5 & 0 & 0 \\
\hline
\end{tabular}

From these examples, it is clear that our proposed PID closely resembles the PID proposed by Ince [14]. This should not come as a surprise because the pointwise approach suggested by Ince expresses associations in terms of mutual information a posteriori.

In this article, we have shown that a partial information decomposition comprising nonnegative unique and redundant contributions follows naturally from the framework of causal tensors. Because we introduced no new information theoretical measures, it is our contention that a partial information decomposition is possible within the framework of "classical" Shannon information theory. The partial information decomposition is problematic when no exact expressions for indirect paths can be determined. It reduces to a rather straightforward exercise when this is possible, for example, within the framework of causal tensors. Two issues that raise some worry about our proposal are: (i) Our proposed definition disagrees with the common opinion that there is redundancy in the AND/OR case. (ii) Our proposed definition does not violate the local positivity property in the case of the system used to prove that local positivity, left monotonicity and identity can not coexist. Future investigations are needed to assess the usefulness of our approach.

Funding: This research received no external funding.

Acknowledgments: I would like to thank Ryan James for asking relevant questions and providing me with challenging examples. 
Conflicts of Interest: The authors declare no conflict of interest.

\section{Appendix A.}

Sketch of Proof of Left Monotonicity Property 2.2.2. Assume that there are two "destinations", $Z_{1}$ and $Z_{1}$. Furthermore, assume that the redundant information in destination $Z_{1} Z_{2}$ equals the mutual information of the path $\left\{x, y,\left(z_{1}, z_{2}\right)\right\}$ :

$$
R\left(Z_{1} Z_{2} ; Y, X\right)=\sum_{i, k_{1}, k_{2}} p^{i, k_{1}, k_{2}} \log _{2}\left[\frac{\sum_{j} A_{i}^{j} B_{j}^{k_{1} k_{2}}}{p^{k_{1} k_{2}}}\right] .
$$

The index $k_{1}$ is related to destination $Z_{1}$ and the index $k_{2}$ is related to destination $Z_{2}$. With the chain rule for probability we get $B_{j}^{k_{1} k_{2}}=B_{j}^{k_{1}} p_{j k_{1}}^{k_{2}}$, and $p^{k_{1} k_{2}}=p^{k_{1}} p_{k_{1}}^{k_{2}}$, equation A1 can be rewritten as

$$
R\left(Z_{1} Z_{2} ; Y, X\right)=\sum_{i, k_{1}, k_{2}} p^{i, k_{1}, k_{2}} \log _{2}\left[\frac{\sum_{j} A_{i}^{j} B_{j}^{k_{1}}}{p^{k_{1}}}\right]+\sum_{i, k_{1}, k_{2}} p^{i, k_{1}, k_{2}} \log _{2}\left[\frac{\sum_{j} A_{i}^{j} B_{j}^{k_{1}} p_{j k_{1}}^{k_{2}}}{\sum_{j} A_{i}^{j} B_{j}^{k_{1}} p_{k_{1}}^{k_{2}}}\right] .
$$

The second sum is always nonnegative because $p_{k_{1}}^{k_{2}}=\sum_{j^{\prime}} p^{j^{\prime}} p_{j^{\prime} k_{1}}^{k_{2}}$ :

$$
R\left(Z_{1} Z_{2} ; Y, X\right) \geq \sum_{i, k_{1}, k_{2}} p^{i, k_{1}, k_{2}} \log _{2}\left[\frac{\sum_{j} A_{i}^{j} B_{j}^{k_{1}}}{p^{k_{1}}}\right] .
$$

The right-hand side equals the mutual information of the path $\left\{x, y, z_{1}\right\}$. Because the proposed redundancy is the minimum mutual information over paths its follows that

$$
R\left(Z_{1} ; Y, X\right) \leq R\left(Z_{1} Z_{2} ; Y, X\right)
$$

\section{Appendix B.}

Sketch of Proof of Identity Property 2.2.3. Per definition

$$
R(X Y ; X, Y)=\min [I\{x, y, x y\}, I\{y, x, x y\}] .
$$

Assume that, in index notation, $p^{i}$ equals the pmf of $X, p^{j}$ equals the $\operatorname{pmf}$ of $Y$, and $p^{i j}$ equals the pmf of $X Y$. Assume furthermore that $A_{i}^{j}$ are the tensor elements for the path $\{x, y\}$. Per definition $A_{j}^{\ddagger i}$ represent the tensor elements for the path $\{y, x\}$.

Let tensor elements for the path $\{x, x y\}$ equal $C_{i^{\prime}}^{i j}$, and the tensor elements for the path $\{y, x y\}$ equal $B_{j^{\prime}}^{i j}$. Using these tensors, the redundancy equals

$$
R(X Y ; X, Y)=\min \left[\sum_{i, j} p^{i j} \log _{2}\left[\frac{\sum_{j^{\prime}} A_{i}^{j^{\prime}} B_{j^{\prime}}^{i j}}{p^{i j}}\right], \sum_{i, j} p^{i j} \log _{2}\left[\frac{\sum_{i^{\prime}} A_{j}^{i^{\prime}} C_{i^{\prime}}^{i j}}{p^{i j}}\right]\right] .
$$

The tensor elements $B_{j^{\prime}}^{i j}$ are related to $A_{j}^{\ddagger i}$. It follows immediately that $B_{j^{\prime}=j}^{i j}=A_{j}^{\ddagger i}$ and $B_{j^{\prime} \neq j}^{i j}=0$, Likewise, $C_{i^{\prime}=i}^{i j}=A_{i}^{j}$ and $C_{i^{\prime} \neq i}^{i j}=0$. We can rewrite the joint probability as $p^{i j}=p^{i} A_{i^{\prime}}^{j}$, and as $p^{i j}=p^{j} A_{j}^{\ddagger i}$. With these expressions, we can rewrite the equation for the redundancy:

$$
R(X Y ; X, Y)=\min \left[\sum_{i, j} p^{i j} \log _{2}\left[\frac{A_{j}^{\ddagger i}}{p^{i}}\right], \sum_{i, j} p^{i j} \log _{2}\left[\frac{A_{i}^{j}}{p^{j}}\right]\right] .
$$


Because both sums represent $I(X ; Y)$, the mutual information between $X$ and $Y$, the proposed redundancy measure satisfies the identity property.

1. Shannon, C.E. A Mathematical Theory of Communication. Bell System Technical Journal, 27, 379-423, [https://onlinelibrary.wiley.com/doi/pdf/10.1002/j.1538-7305.1948.tb01338.x]. doi:10.1002/j.1538-7305.1948.tb01338.x.

2. McGill, W. Multivariate information transmission. Psychometrika 1954, 19, 97-116.

3. Williams, P.; Beer, R. Nonnegative Decomposition of Multivariate Information. preprint 2010, 1004.

4. Lizier, J.; Bertschinger, N.; Wibral, M. Information Decomposition of Target Effects from Multi-Source Interactions: Perspectives on Previous, Current and Future Work. Entropy 2018, 20, 307. doi:10.3390/e20040307.

5. Cover, T.M.; Thomas, J.A. Elements of Information Theory; Wiley-Interscience: New York, NY, USA, 1991.

6. Papoulis, A.; Pillai, S.U. Probability, Random Variables, and Stochastic Processes, fourth ed.; McGraw Hill, 2002.

7. Schreiber, T. Measuring Information Transfer. Phys. Rev. Lett. 2000, 85, 461-464. doi:10.1103/PhysRevLett.85.461.

8. Beckenbach, E.F. Modern mathematics for the engineer: second series; New York : McGraw-Hill, 1961.

9. Wibral, M.; Pampu, N.; Priesemann, V.; Siebenhühner, F.; Seiwert, H.; Lindner, M.; Lizier, J.T.; Vicente, R. Measuring information-transfer delays. PloS one 2013.

10. Sigtermans, D. Towards a Framework for Observational Causality from Time Series: When Shannon Meets Turing, 2020, [202001.0106]. doi:10.20944/preprints202001.0106.v1.

11. James, R.G.; Barnett, N.; Crutchfield, J.P. Information Flows? A Critique of Transfer Entropies. Physical Review Letters 2016, 116. doi:10.1103/physrevlett.116.238701.

12. Bertschinger, N.; Rauh, J.; Olbrich, E. Shared Information - New Insights and Problems in Decomposing Information in Complex Systems. Springer Proceedings in Complexity 2012. doi:10.1007/978-3-319-00395-5_35.

13. Harder, M.; Salge, C.; Polani, D. Bivariate measure of redundant information. Physical review. E, Statistical, nonlinear, and soft matter physics 2013, 87, 012130. doi:10.1103/PhysRevE.87.012130.

14. Ince, R. Measuring Multivariate Redundant Information with Pointwise Common Change in Surprisal. Entropy 2016, 19. doi:10.3390/e19070318.

15. Rauh, J.; Bertschinger, N.; Olbrich, E. Reconsidering unique information: Towards a multivariate information decomposition. IEEE International Symposium on Information Theory-Proceedings 2014, arXiv. doi:10.1109/ISIT.2014.6875230.

16. James, R.; Crutchfield, J. Multivariate Dependence Beyond Shannon Information. Entropy 2016, 19. doi:10.3390/e19100531.

17. Bertschinger, N.; Rauh, J.; Olbrich, E.; Ay, N. Quantifying Unique Information. Entropy 2013, 16. doi:10.3390/e16042161. 